Mario F. Mendez, MD, $\mathrm{PhD}$

Aditi Joshi, PhD

Kanida Tassniyom, MD Edmond Teng, MD, PhD Jill S. Shapira, RN, PhD

Correspondence to Dr. Mendez: mmendez@ucla.edu

Supplemental data at www.neurology.org

\section{Clinicopathologic differences among patients with behavioral variant frontotemporal dementia}

\section{[메}

\section{ABSTRACT}

Objective: To characterize the presenting symptoms and signs of patients clinically diagnosed with behavioral variant frontotemporal dementia (bvFTD) and who had different neuropathologic findings on autopsy.

Methods: This study reviewed all patients entered as clinical bvFTD in the National Alzheimer's Coordinating Center's database and who had both clinical and neuropathologic data from 2005 to 2011. Among the 107 patients identified, 95 had unambiguous pathologic findings, including 74 with frontotemporal lobar degeneration (bvFTD-FTLD) and 21 with Alzheimer disease (bvFTD-AD). The patients with bvFTD-FTLD were further subdivided into $\tau$-positive $(n=23)$ or $\tau$-negative $(n=51)$ histopathology subgroups. Presenting clinical signs and symptoms were compared between these neuropathologic groups.

Results: The patients with bvFTD-FTLD were significantly more likely than patients with bvFTD-AD to have initially predominant personality changes and poor judgment/decision-making. In contrast, patients with bvFTD-AD were more likely than patients with bvFTD-FTLD to have memory difficulty and delusions/hallucinations and agitation. Within the bvFTD-FTLD group, the $\tau$-positive subgroup had more patients with initial behavioral problems and personality change than the $\tau$-negative subgroup, who, in turn, had more patients with initial cognitive impairment and speech problems.

Conclusion: During life, patients with AD pathology may be misdiagnosed with bvFTD if they have an early age at onset and prominent neuropsychiatric features despite having greater memory difficulties and more intact personality and executive functions than patients with bvFTD-FTLD. Among those with FTLD pathology, patients with $\tau$-positive bvFTD were likely to present with behavior/personality changes. These findings offer clues for antemortem recognition of neuropathologic subtypes of bvFTD. Neurology ${ }^{\circledR}$ 2013;80:561-568

\section{GLOSSARY}

$\mathbf{A D}=$ Alzheimer disease; $\mathbf{A D C}=$ Alzheimer Disease Centers; bvFTD $=$ behavioral variant frontotemporal dementia; $\mathbf{C B D}=$ corticobasal degeneration; FTLD = frontotemporal lobar degeneration; FUS = fused in sarcoma; MMSE = Mini-Mental State Examination; NACC $=$ National Alzheimer's Coordinating Center; $\mathbf{N I A}=$ National Institute of Aging; $\mathbf{N P I} \mathbf{Q}=$ Neuropsychiatric Inventory-Questionnaire; PSP $=$ progressive supranuclear palsy; TDP-43 $=\tau$ or transactive response DNA-binding protein of 43 kD; UDS = Uniform Data Set; UPDRS = Unified Parkinson's Disease Rating Scale.

Behavioral variant frontotemporal dementia (bvFTD) is a common dementia among those with an age at onset of 65 years or younger. ${ }^{1}$ Only patients meeting clinical criteria for Alzheimer disease (AD) are more common than bvFTD among those with early-onset neurodegenerative dementias. bvFTD is associated with disinhibition, apathy, loss of empathy, compulsive or stereotypical behavior, dietary changes, and a dysexecutive neuropsychological profile. ${ }^{2,3}$ There is no definitive antemortem test for bvFTD, and diagnosis depends on clinical criteria which have been validated by clinicopathologic correlation. ${ }^{2,4,5}$ Clinical criteria, however, are not infallible, and some patients diagnosed with bvFTD can still have $\mathrm{AD}$ or another disorder on autopsy. ${ }^{6}$

This investigation examined whether different underlying neuropathologic findings in clinically diagnosed bvFTD were associated with different presenting signs and symptoms. We analyzed

From the Departments of Neurology (M.F.M., A.J., E.T., J.S.S.) and Psychiatry \& Biobehavioral Sciences (M.F.M.), David Geffen School of Medicine, University of California at Los Angeles, Los Angeles; VA Greater Los Angeles Healthcare Center (M.F.M., E.T.), Los Angeles, CA; and Department of Psychiatry (K.T.), Khon Kaen University, Khon Kaen, Thailand.

Go to Neurology.org for full disclosures. Funding information and disclosures deemed relevant by the authors, if any, are provided at the end of the article. 
participants included in the National Alzheimer's Coordinating Center (NACC) database who were clinically diagnosed with bvFTD and underwent autopsy evaluations. ${ }^{7-9}$ A significant proportion of these patients had predominately the neuropathology of $\mathrm{AD} .{ }^{10}$ Therefore, we compared the clinical features of patients with bvFTD with the expected frontotemporal lobar degeneration (FTLD) on neuropathology to those with $\mathrm{AD}$ on neuropathology. We subsequently compared the clinical features of patients with bvFTD in the subgroup with FTLD who were $\tau$-positive to those who were $\tau$-negative on neuropathology. The results of this investigation expand on the reasons for misdiagnosis of bvFTD across a cross-section of clinicians and medical centers and contribute to the antemortem differentiation of those with FTLD who have $\tau$-positive vs $\tau$-negative pathology.

METHODS Participants. This study accessed data from all patients included in the NACC neuropathology dataset with a clinical diagnosis of dementia. This subset of the NACC neuropathologic data was cross-referenced with clinical information abstracted from the NACC Uniform Data Set (UDS). ${ }^{7,8}$ The final dataset analyzed for the current study included 1,215 subjects evaluated at National Institute on Aging-funded Alzheimer Disease Centers (ADCs) between 2005 and 2011. The NACC database includes 34 past and present ADCs.

Standard protocol approvals, registrations, and patient consents. This study was approved by the Institutional Review Board at the University of California at Los Angeles.

Part A. Among the 1,215 patients with dementia with autopsies from 2005 to July 2011, we identified 107 with a primary clinical diagnosis of bvFTD. This study was limited to the behavioral variant of FTD and did not include the language variants. Most patients were enrolled from 13 centers with a interest in non- $\mathrm{AD}$ dementias. These well-studied patients were primarily diagnosed with the 1998 Consensus Criteria for bvFTD (the new International Consensus Criteria postdate these assessments) ${ }^{2,4}$ For participants with multiple visits, clinical data from the earliest visit were used in our analyses. Of the 107 patients, 12 proved to have contradictory or ambiguous neuropathologic data, including uncertainty regarding predominant pathology or conflicting information regarding $\tau$ findings, and were eliminated from all subsequent analyses. The remaining patients were subdivided into those having predominantly FTLD neuropathology (bvFTD-FTLD group) and those having predominantly non-FTLD pathology.

Of the 21 patients having predominantly non-FTLD pathology, all proved to have $\mathrm{AD}$ pathology (bvFTD-AD group) by National Institute of Aging (NIA)-Reagan Criteria. ${ }^{10}$ Twenty had a "high likelihood of dementia being due to $\mathrm{AD}$," and 1 had an "intermediate likelihood of dementia being due to $\mathrm{AD}$." None of these 21 patients had neocortical Lewy bodies, a "primary diagnosis of Lewy body," or other significant pathology. Therefore, part A of this study compared the bvFTD-FTLD group $(\mathrm{n}=74)$ with the bvFTD-AD group $(\mathrm{n}=$ 21) on UDS clinical variables in 3 domains: cognitive, behavior, and motor.
Part B. The 74 patients with bvFTD-FTLD were further characterized based on the presence of $\tau$-positive or $\tau$-negative pathology. There were multiple overlapping neuropathologic categories such that patients classified as " $\tau$-positive" had the following designations: Pick disease $(n=4)$, progressive supranuclear palsy $(n=9)$, corticobasal degeneration (5), FTD and parkinsonism with $\tau$-positive or argyrophilic inclusions $(n=9)$, and other tauopathy $(n=1)$. There were no specific data on the reliability of any of these categories, and patients could have more than one category within the $\tau$-positive groupings, or have changes suggestive of corticobasal degeneration (CBD) or progressive supranuclear palsy (PSP). Patients classified as " $\tau$-negative" had the following pathologic designations: FTD with ubiquitinpositive ( $\tau$-negative) inclusions $(\mathrm{n}=47)$ and FTD with no distinctive histopathology ( $\tau$-negative, ubiquitin-negative, and no argyrophilic inclusions) $(n=4)$. Part B of this study compared the patients with bvFTD-FTLD in the $\tau$-positive group $(\mathrm{n}=23$ ) with the patients with bvFTD-FTLD in the $\tau$-negative group $(\mathrm{n}=51)$ on UDS clinical variables in the 3 domains: cognitive, behavior, and motor.

Clinical assessments. The clinical variables involved presenting symptoms for cognition, behavior, and motor based on form B9 from the initial visit. This was completed by the clinician, with conclusions based on information obtained through the subject, informants, and medical records or observation. The categories included predominant domain which was first recognized as changed in the subject (first domain), the predominant symptom which was first recognized as a decline in the subject's cognition/behavioral symptoms/motor symptoms (first symptoms), and whether the subject currently had cognitive/behavioral/motor symptoms (current symptoms) (www.alz. washington.edu/NONMEMBER/UDS/DOCS/VER2/ivpguide.pdf). These results were compared with the corresponding intake assessment measures: neuropsychological testing, Neuropsychiatric InventoryQuestionnaire (NPI-Q), ${ }^{11}$ and the neurologic examination. For the neurologic examination, this study included the brief examination that is part of the UDS. In addition, the Unified Parkinson's Disease Rating Scale (UPDRS) was available for inclusion as part of the neurologic examination. ${ }^{12}$ Given the prevalence of apraxia of speech in FTLD, the speech abnormality section of the scale was further extracted as a separate examination item.

Statistical analyses. All statistical analyses were performed with SPSS version 19 (SPSS, Chicago, IL). Comparisons between neuropathologic groups were performed using $t$ tests for demographic, and approximately normally distributed, continuous variables and $\chi^{2}$ for the clinicopathologic, categorical variables. Subjects with missing or unknown data were excluded from analysis except where absence of mention of prominent individual signs or symptoms could be reasonably imputed as absence of those signs or symptoms.

RESULTS Demographic data were similar across all 3 bvFTD neuropathologic subgroups (table 1). There were no significant differences in gender distribution; years of formal education; Mini-Mental State Examination (MMSE) scores; ethnicity; mean ages at symptom onset, clinical presentation, or death; or proportion of subjects reporting a first-degree relative diagnosed with dementia.

Comparison of bvFTD-FTLD vs bvFTD-AD neuropathologic groups. On initial visit, the 2 groups did not differ based on first domain affected, although the patients with bvFTD-AD trended to more subjects with involvement of the cognitive domain $(\mathrm{n}=35,47.3 \%$ vs $\mathrm{n}=14$, $67.7 \% ; \chi^{2}=1.73$, NS). Similar proportions of subjects (bvFTD-FTLD vs bvFTD-AD) had first predominant 


\begin{tabular}{|c|c|c|c|}
\hline \multirow[t]{2}{*}{ frontotemporal dem } & $\begin{array}{l}\text { f patients wi } \\
\text { la with neuro }\end{array}$ & $\begin{array}{l}\text { ehavioral var } \\
\text { ology }^{\text {a }}\end{array}$ & \multirow[b]{2}{*}{$\begin{array}{l}\text { bvFTD-AD } \\
(\mathrm{n}=21)\end{array}$} \\
\hline & $\begin{array}{l}\tau \text {-Negative } \\
(\mathrm{n}=51)\end{array}$ & $\begin{array}{l}\tau \text {-Positive } \\
(n=23)\end{array}$ & \\
\hline Male, n (\%) & $35(68.6)$ & $14(60.8)$ & $18(85.7)$ \\
\hline Mean education, $y$, mean \pm SD & $15.15 \pm 2.9$ & $15.32 \pm 3.2$ & $16.25 \pm 3.4$ \\
\hline Presentation MMSE, mean \pm SD & $18.2 \pm 9.3$ & $18.3 \pm 9.7$ & $13.3 \pm 9.4$ \\
\hline Caucasian, n (\%) & $50(98.0)$ & $22(95.7)$ & $18(85.7)$ \\
\hline Age at onset, $y$, mean $\pm S D$ & $59.3 \pm 9.9$ & $59.2 \pm 8.2$ & $63.2 \pm 7.7$ \\
\hline Age at presentation, $y$, mean $\pm S D$ & $64.2 \pm 9.5$ & $65.3 \pm 7.9$ & $69.3 \pm 8.3$ \\
\hline Age at death, $y$, mean \pm SD & $65.5 \pm 10.2$ & $67.3 \pm 7.3$ & $70.5 \pm 7.2$ \\
\hline Family history of dementia, $\mathrm{n}(\%)^{\mathrm{b}}$ & 15 (29.4) & $11(47.8)$ & 11 (52.3) \\
\hline
\end{tabular}

Abbreviations: $A D=$ Alzheimer disease; bvFTD = behavioral variant frontotemporal dementia; MMSE $=$ Mini-Mental State Examination

${ }^{\text {a }}$ No differences were significant.

${ }^{b}$ Number of cases with one or more first-degree relatives with dementia. changes in behavior domain $(\mathrm{n}=35,47.3 \%$ vs $\mathrm{n}=7$, $\left.33.3 \% ; \chi^{2}=0.79, \mathrm{NS}\right)$ and motor domain $(\mathrm{n}=4$, $5.4 \%$ vs $0 ; \chi^{2}=0.22$, NS).

First and current symptoms. There were more memory deficits and personality changes in the bvFTD-AD group compared to more executive (judgment/problem-s olving) deficits in the bvFTD-FTLD group (figure 1 and table 2). Significantly more patients with bvFTD$\mathrm{AD}(\mathrm{n}=8,38.1 \%)$ had first (but not current) cognitive symptoms of memory deficits compared to patients with bvFTD-FTLD ( $\mathrm{n}=10,13.5 \%$; $\left.\chi^{2}=4.94, p<0.05\right)$, whereas significantly more patients with bvFTD-FTLD ( $\mathrm{n}=44,59.5 \%$ ) had first cognitive symptoms of judgment/problem-solving deficits compared to patients with bvFTD-AD ( $\mathrm{n}=5,23.8 \%$; $\left.\chi^{2}=6.96, p<0.01\right)$. The groups did not differ on other cognitive measures. On first and current behavioral symptoms, patients with

Figure 1 Behavioral variant frontotemporal dementia-frontotemporal lobar degeneration vs behavioral variant frontotemporal dementia-Alzheimer disease neuropathologic groups: First symptoms

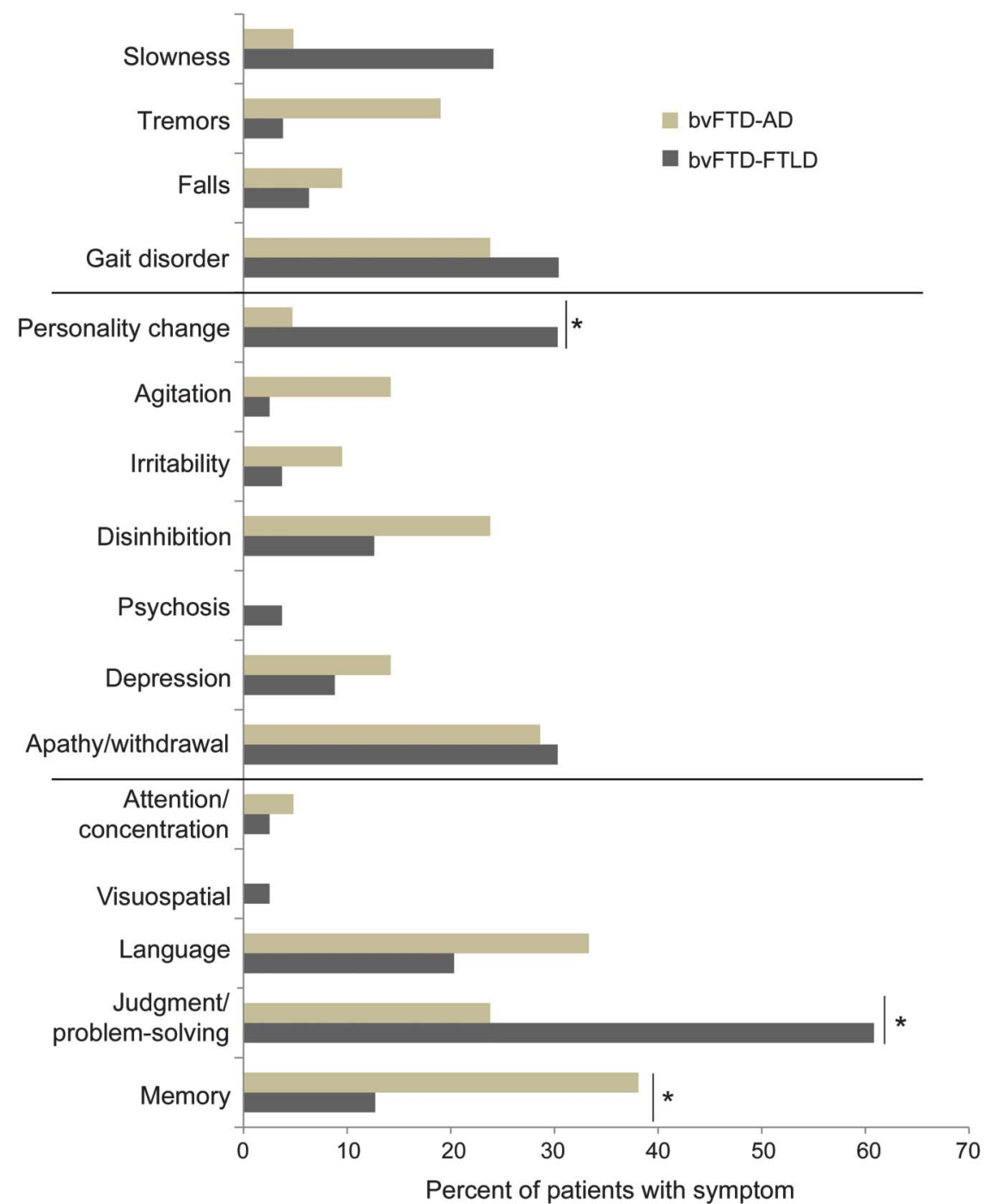

$A D=$ Alzheimer disease; bvFTD = behavioral variant frontotemporal dementia; FTLD = frontotemporal lobar degeneration. *Significant differences between the groups. 


\begin{tabular}{|c|c|c|c|c|c|c|}
\hline \multicolumn{7}{|c|}{ Table 2 Current symptoms among neuropathologic groups } \\
\hline Symptoms & bvFTD-FTLD, n (\%) & bvFTD-AD, n (\%) & Significance $\left(\chi^{2}\right)$ & $\tau$-Positive, $\mathrm{n}(\%)$ & $\tau$-Negative, $\mathrm{n}(\%)$ & Significance $\left(x^{2}\right)$ \\
\hline Memory & 65 (87.8) & $21(100)$ & NS & 20 (86.9) & 45 (88.2) & NS \\
\hline Judgment and problem-solving & 73 (98.6) & $21(100)$ & NS & $23(100)$ & $50(98.0)$ & NS \\
\hline Language & 66 (89.2) & $18(85.7)$ & NS & 19 (82.6) & $47(92.1)$ & NS \\
\hline Visuospatial & $40(54.1)$ & $16(76.2)$ & NS & $11(47.8)$ & 19 (37.3) & NS \\
\hline Attention/concentration & 57 (77.0) & $18(85.7)$ & NS & 18 (78.2) & $39(76.4)$ & NS \\
\hline Apathy/withdrawal & $54(73.0)$ & $15(71.4)$ & NS & 17 (73.9) & 37 (72.5) & NS \\
\hline Depression & 32 (43.2) & $12(57.1)$ & NS & $10(43.5)$ & $22(43.1)$ & NS \\
\hline Psychosis & 11 (14.9) & $6(28.5)$ & NS & $4(17.3)$ & 7 (13.7) & NS \\
\hline Disinhibition & 49 (66.2) & $14(66.7)$ & NS & 17 (73.9) & $32(62.7)$ & NS \\
\hline Irritability & 27 (36.5) & $13(61.9)$ & NS & $8(34.7)$ & 19 (37.2) & NS \\
\hline Agitation & 25 (33.8) & $12(57.1)$ & NS & $7(30.4)$ & 18 (35.3) & NS \\
\hline Personality change & 58 (78.3) & $11(52.4)$ & $4.33 ; p<0.05$ & 18 (78.2) & $40(78.4)$ & NS \\
\hline Gait disorder & 39 (52.7) & 7 (33.3) & NS & 9 (39.1) & $30(58.8)$ & NS \\
\hline Falls & $24(32.4)$ & 7 (33.3) & NS & $6(26.1)$ & 18 (35.3) & NS \\
\hline Tremors & $21(28.4)$ & 8 (38.1) & NS & $6(26.1)$ & $15(29.4)$ & NS \\
\hline Slowness & $37(50)$ & 8 (38.1) & NS & $10(43.5)$ & 27 (52.9) & NS \\
\hline
\end{tabular}

Abbreviations: $A D=$ Alzheimer disease; bvFTD = behavioral variant frontotemporal dementia; FTLD = frontotemporal lobar degeneration.

bvFTD-FTLD were more likely to develop a significant personality change (first symptoms: $\mathrm{n}=21,28.4 \%$, and current symptoms: $\mathrm{n}=58,78.3 \%)$ compared to patients with bvFTD-AD (first symptoms: $\mathrm{n}=1,4.7 \%$; $\chi^{2}=3.98, p<0.05$, and current symptoms: $\mathrm{n}=11$, $\left.52.4 \% ; \chi^{2}=4.33, p<0.05\right)$. There were no group differences on first or current motor symptoms.

Assessment measures. There were no group differences on neuropsychological tests or the neurologic examination (tables e- 1 and e-2 on the Neurology ${ }^{\circledR}$ Web site at www.neurology.org), but, in contrast to the greater personality changes among the patients with bvFTDFTLD, the patients with bvFTD-AD were more likely to have behavioral abnormalities on the NPI-Q (figure 2). Delusions and hallucinations were observed in significantly more of the patients with bvFTD-AD $(n=8$, $38.1 \%$ and $\mathrm{n}=7,33.3 \%$ ) as compared to the patients with bvFTD-FTLD ( $\mathrm{n}=11,14.9 \% ; \chi^{2}=4.16, p<$ 0.05 and $\left.n=6,8.1 \% ; \chi^{2}=6.81, p<0.01\right)$. Agitation was also observed in significantly more of the patients with bvFTD-AD ( $\mathrm{n}=16,76.2 \%)$ as compared to the patients with bvFTD-FTLD ( $\mathrm{n}=33,44.6 \% ; \chi^{2}=$ $5.34, p<0.05$ ). When severity of agitation was noted, it was most often rated "severe" among the patients with bvFTD-AD $(\mathrm{n}=5,55.5 \%)$ as compared to the patients with bvFTD-FTLD $\left(\mathrm{n}=5,6.7 \%\right.$; $\chi^{2}=12.9, p<$ 0.001).

Comparison of bvFTD-FTLD $\tau$-positive vs bvFTD-FTLD $\tau$-negative neuropathologic groups. For first domain, the $\tau$-negative group had significantly more changes in the cognition domain $(n=28,54.9 \%$ vs $n=6,26.1 \%$ for $\tau$-negative group; $\left.\chi^{2}=4.20, p<0.05\right)$. In contrast, it was the $\tau$-positive group that had significantly more changes in the behavior domain $(\mathrm{n}=17,73.9 \%$ vs $\mathrm{n}=19,37.2 \%$ for $\tau$-negative group; $\chi^{2}=7.12, p<$ 0.01). For motor domain, there were no differences between $\tau$-positive $(n=0)$ and $\tau$-negative $(n=4$, $7.8 \%)$ groups $\left(\chi^{2}=0.68, \mathrm{NS}\right)$.

First and current symptoms. There were no differences in first or current cognitive symptoms or first or current motor symptoms, but there were group differences in behavior (figure 3). For first behavioral symptoms (but not current behavioral symptoms), more $\tau$-positive patients had first-predominant personality changes ( $\mathrm{n}=11,47.8 \%)$ compared to $\tau$-negative patients $\left(\mathrm{n}=10,19.6 \% ; \chi^{2}=4.90, p<0.05\right)$ (figure 3$)$.

Assessment measures. On the neurologic examination, the $\tau$-negative patients $(\mathrm{n}=36,70.6 \%)$ were found to have more speech abnormalities than the $\tau$-positive patients $\left(n=6,28.6 \% ; \chi^{2}=9.15, p<0.01\right)$. There were no differences on neuropsychological tests, NPI-Q (figure e-1), or the rest of the neurologic examination.

DISCUSSION This study accessed patients with clinically well-diagnosed bvFTD in the NACC database, who had presenting clinical information and subsequently neuropathologic assessments. Among those with the clinical diagnosis of bvFTD, the initial presenting clinical symptoms and signs varied depending on whether the underlying neuropathology was $\mathrm{AD}$, FTLD with $\tau$-positive neuronal inclusions, or FTLD with $\tau$-negative findings. These findings revealed reasons 
Figure 2 Frontotemporal lobar degeneration vs Alzheimer disease neuropathologic groups: Neuropsychiatric Inventory Questionnaire

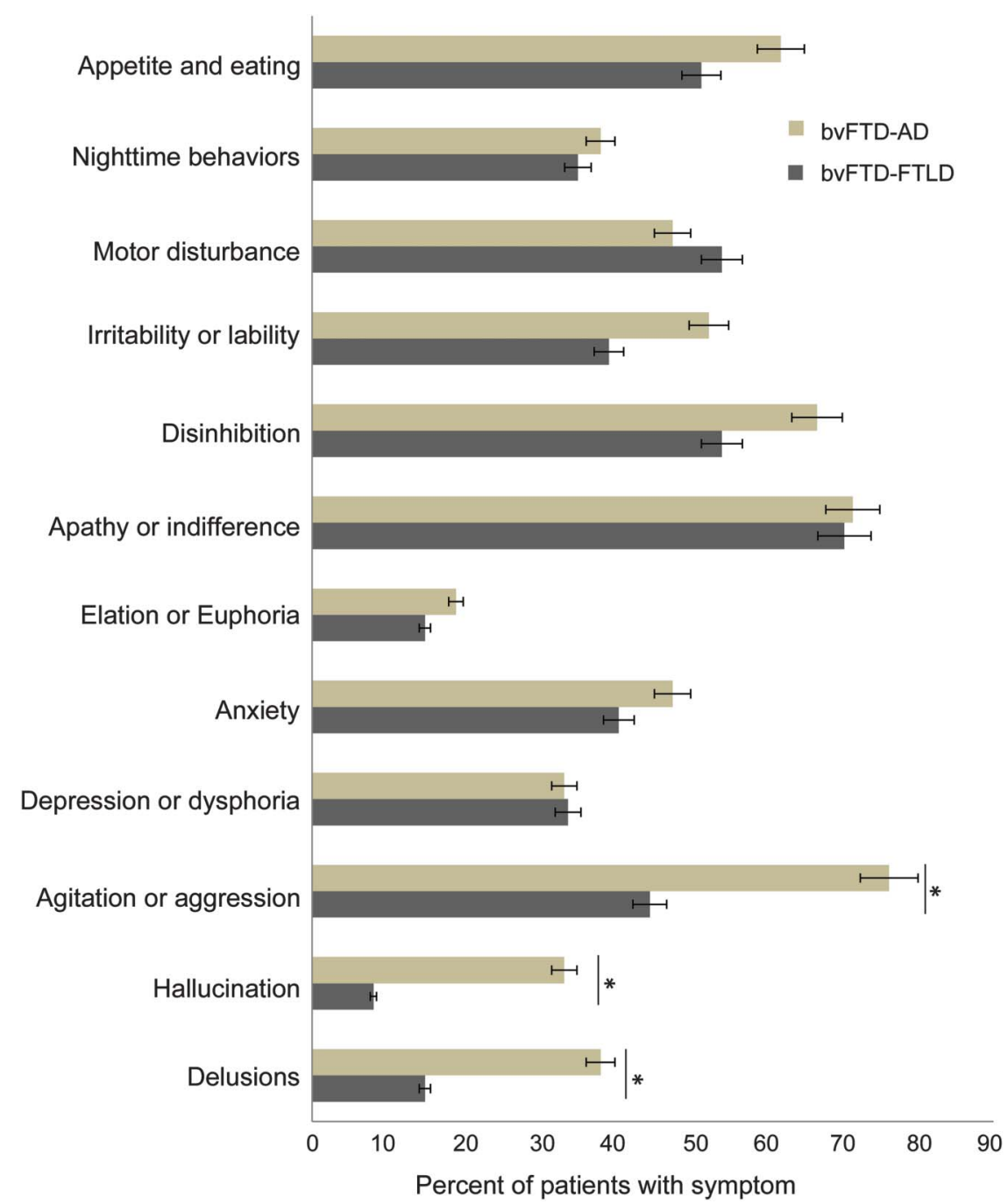

$A D=$ Alzheimer disease; bvFTD = behavioral variant frontotemporal dementia; FTLD = frontotemporal lobar degeneration. *Significant differences between the groups.

why clinicians across different medical centers misdiagnose bvFTD and suggested differentiating clinical features for $\tau$-positive vs $\tau$-negative FTLD.

Despite published and validated diagnostic criteria for bvFTD, ${ }^{2,4}$ clinicians continue to misdiagnose patients with early-onset $\mathrm{AD}$ as having bvFTD. In this study, the neuropathology of $\mathrm{AD}$ is present in about 1 out of every 5 clinically diagnosed bvFTD cases, which is compatible with the results from other series. ${ }^{6,13,14}$ In a major report of 114 cases of bvFTD, there are 19 (16.7\%) with AD on autopsy via Consortium to Establish a Registry for Alzheimer's Disease criteria. ${ }^{6,15}$ That report, unlike this one, is not a prevalence study because it combined groups with FTLD pathology and with clinical FTD with non-FTLD pathology. Other investigators report $\mathrm{AD}$ pathology in $12 \%$ of a small cohort of patients with bvFTD, ${ }^{14}$ and AD pathology via NIA/ Reagan criteria with or without neocortical Lewy bodies in 20\% of another cohort of patients with bvFTD. ${ }^{13}$ Similar to our study, patients with bvFTD and FTLD pathology have been less likely to present with cognitive complaints when compared with the subgroup with AD pathology, whereas those with $\mathrm{AD}$ pathology have been more likely to present with cognitive difficulties, particularly impairments in episodic memory. ${ }^{6}$ Also similar to our study, others have found that over $70 \%$ of patients with bvFTD have poor planning and lack of judgment ${ }^{16}$ The results reported here add to the existing literature by indicating that patients with $\mathrm{AD}$ neuropathology may be clinically misdiagnosed with bvFTD when they are of early onset and have prominent neuropsychiatric features (delusions, hallucinations, agitation) despite greater memory difficulty and more intact personality and executive functions. In addition, clinicians may confuse the frontal clinical variant of $\mathrm{AD}$ with executive deficits as bvFTD. ${ }^{17}$ 
Figure 3 Frontotemporal lobar degeneration $\tau$-positive vs frontotemporal lobar degeneration $\tau$-negative neuropathologic subgroups: First symptoms

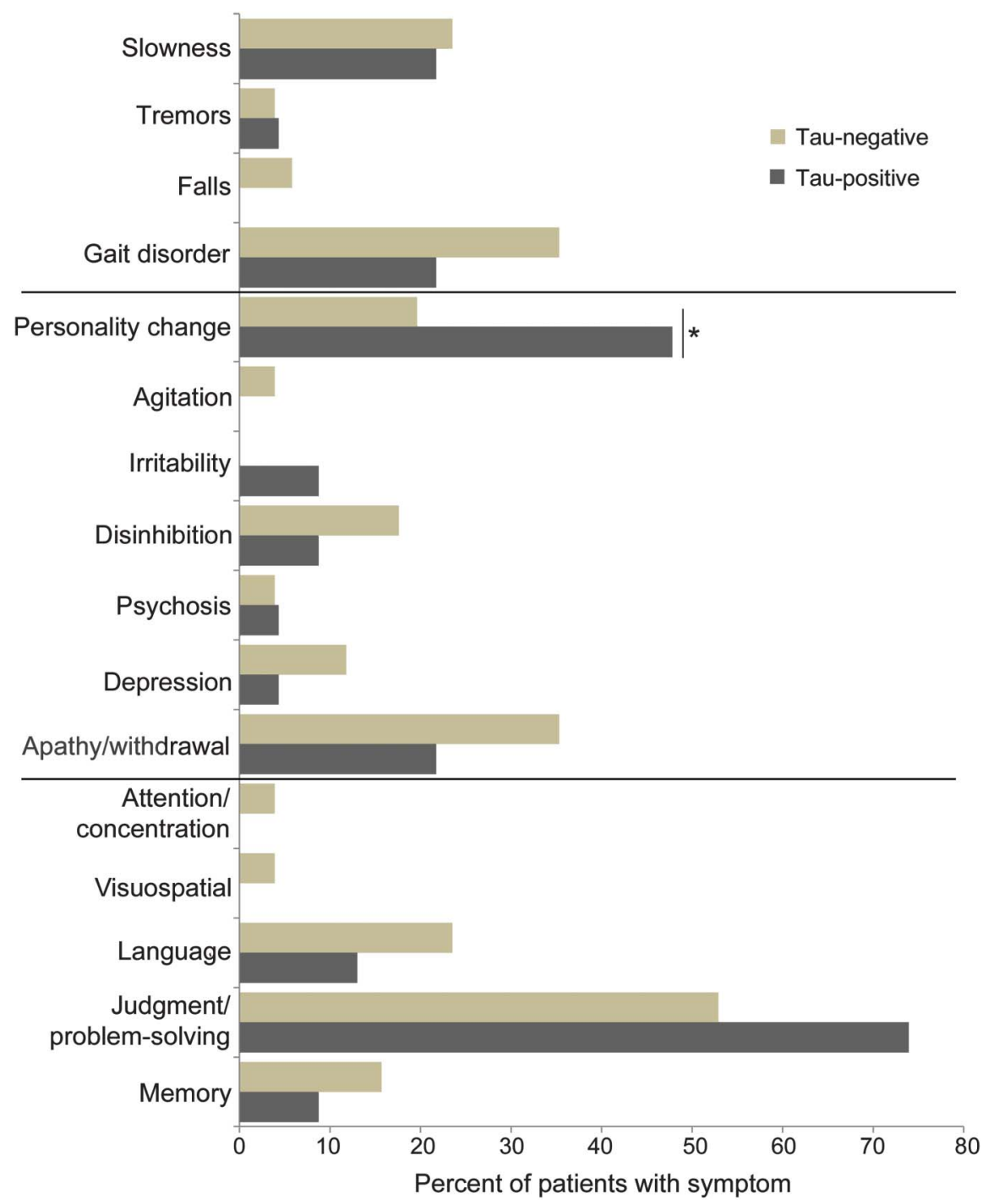

*Significant differences between the groups.

Neuropathologists commonly characterize FTLD by intraneuronal inclusions containing 1 of 2 misfolded, hyperphosphorylated, and ubiquinated proteins, $\tau$ or transactive response DNA-binding protein of $43 \mathrm{kD}$ (TDP-43). ${ }^{18}$ Most of the $\tau$-negative, ubiquitin-positive patients have TDP-43, although a smaller percentage have inclusions with fused in sarcoma (FUS) or no inclusions at all. ${ }^{19}$ During life, it has not been possible to reliably distinguish those patients with bvFTD who have underlying $\tau$-negative or $\tau$-positive pathology without the benefit of brain biopsy. Yet, determining the underlying pathology, whether $\tau$-positive or $\tau$-negative FTLD, or another pathology such as $\mathrm{AD}$, may become increasingly important with the potential development of rational drug therapies targeted to specific pathologic conditions.

Estimates vary as to the proportions of $\tau$-positive vs $\tau$-negative pathology among patients with bvFTD. Of approximately 128 patients with bvFTD reported in a pooled study, $58(45 \%)$ are $\tau$-positive and $70(55 \%)$ are $\tau$-negative, mostly with TDP-43..$^{3,13,20-22}$ Among the $\tau$-positive cases in this pooled sample, the neuropathology of Pick disease occurs in about $70 \%$ of cases, with CBD in another 20\%, and PSP in many of the remainder. ${ }^{3,6,13,20-22}$ In our study, we could not tell the percentages of the individual $\tau$ pathologies; there are overlapping categories, the classification of $17.4 \%$ with Pick disease does not reflect the true percentage of Pick disease pathology, and there is more PSP pathology than usually reported. Moreover, in the subset of the NACC neuropathology dataset analyzed here, there is a relatively smaller proportion of $\tau$-positive cases $(31.1 \%)$ and a correspondingly larger proportion of $\tau$-negative cases $(68.9 \%)$.

Distinguishing $\tau$-positive from $\tau$-negative patients during life can be difficult, primarily because the clinical 
manifestations depend more on the neuroanatomical localization than on type of neuronal inclusions. ${ }^{23}$ Some investigators report that, compared to $\tau$-negative patients, $\tau$-positive patients tend to have an extrapyramidal disorder suggestive of CBD, PSP, or parkinsonism, ${ }^{6}$ which would indicate that extrapyramidal changes on motor examination might predict an underlying tauopathy. However, $\tau$-negative patients could also have extrapyramidal changes, and the current NACC series fails to reveal consistent differences in motor findings or total UPDRS scores. ${ }^{13,21,22,24}$ One study indicates a particular tendency to executive problems in planning and judgment among $\tau$-positive patients. ${ }^{25}$ In contrast, other studies report greater social or behavioral changes, impaired regulation of personal conduct, poor personal hygiene, and apathy among $\tau$-negative patients. ${ }^{6,25,26}$ Our finding of a greater proportion of $\tau$-positive patients with initial behavioral and personality changes is seemingly in contradiction with these reports. The personality changes in the NACC are informant responses to whether the patients exhibited uncharacteristic as well as bizarre behavior, and this could reflect a general change in comportment from any cause, particularly since $\tau$-positive patients tend to exhibit more advanced prefrontal-temporal atrophy on presentation than do $\tau$-negative patients. ${ }^{27}$ Consistent with our findings, there may be greater abnormalities in speech and cognition, including language, among $\tau$-negative patients. ${ }^{6,26}$ Clearly much more work is needed in order to establish the nature of potential clinical differences between patients with bvFTD who have $\tau$-positive or $\tau$-negative FTLD pathology.

This study had several potential limitations. First, although the NACC UDS and neuropathology datasets archive data from a large number of participants, data collection was performed by multiple clinicians and neuropathologists at different research centers, raising the possibility of variability in diagnostic interpretation across centers. However, NACC evaluations were standardized and reliability training was required across sites. Furthermore, the concordance of the NACC findings with prior single-site studies reinforced the generalizability of our results. Second, the NACC database lacked detailed neuropathologic information on localization of pathology and on histopathologic subtyping of $\tau$, TDP-43, and FUS. Nevertheless, all of the cases included in this study were either $\tau$-positive or $\tau$-negative, the focus of this report. Third, given the limited overlap in subjects between the NACC UDS and neuropathology datasets, and the exploratory nature of our analyses, we did not adjust for multiple comparisons, and missing data for salient signs and symptoms was interpreted as negative results. The number of comparisons per domain and category, however, were all 12 or less, thus mitigating the effects of multiple comparisons, and the number of missing values for the analyzed variables was either none or a few per variable without a clear bias for any group. Finally, much of the data were informant-derived and influenced by their interpretation of questions such as "personality change." Nevertheless, the informants were usually the primary caregivers who know the patients the best and who could most reliably report on their earliest symptomatic changes.

Among patients with clinically diagnosed bvFTD, there are differences in signs and symptoms at presentation between neuropathologically defined subgroups. Patients clinically diagnosed with bvFTD may have $\mathrm{AD}$ pathology, rather than FTLD pathology, if they have an early age at onset and prominent neuropsychiatric features despite greater memory difficulties and relatively intact personality and executive functions. Conversely, clinically diagnosed bvFTD is most likely to be caused by FTLD pathology if patients have early executive dysfunction and personality changes. Although this study did not include the new, more sensitive International Consensus Criteria for bvFTD, those criteria have unclear specificity for distinguishing bvFTD-FTLD from bvFTD-AD, particularly in the presence of early neuropsychiatric features. ${ }^{2}$ In this series, among those with FTLD pathology, patients with $\tau$-positive bvFTD tend to earlier behavioral and personality changes, and $\tau$-negative patients tend to more cognitive and speech abnormalities. These findings offer clues to recognition of pathologic subtypes of bvFTD during life, an important step for the development of pathophysiologically targeted clinical drug trials.

\section{AUTHOR CONTRIBUTIONS}

Dr. Mendez: study concept and design, analysis and interpretation, revision of manuscript, and study supervision. Dr. Joshi: acquisition of data and analysis and interpretation. Dr. Tassniyom: acquisition of data. Dr. Shapira: study concept and design and revision of manuscript for intellectual content. Dr. Teng: critical revision of the manuscript for important intellectual content.

\section{STUDY FUNDING}

Supported by NIA grant \#R01AG034499-03 (M.F. Mendez), a VA Merit Review (A. Joshi, M.F. Mendez), Alzheimer's Disease Research Center Grant NIA P50 AG-16570 (M.F. Mendez), and the NACC grant (UO1 AG016976).

\section{DISCLOSURE}

M. Mendez received compensation for individual speaking engagements, as an editor for UpToDate, and for book royalties. He has also participated in a clinical drug trial for Forest Pharmaceuticals. A. Joshi and K. Tassniyom report no disclosures. E. Teng reports compensation for serving as a consultant to NeuroVision Imaging. J. Shapira received compensation for participation in a clinical drug trial for Forest Pharmaceuticals. Go to Neurology.org for full disclosures.

Received August 20, 2012. Accepted in final form October 4, 2012.

\section{REFERENCES}

1. Ratnavalli E, Brayne C, Dawson K, Hodges JR. The prevalence of frontotemporal dementia. Neurology 2002;58: 1615-1621. 
2. Rascovsky K, Hodges JR, Knopman D, et al. Sensitivity of revised diagnostic criteria for the behavioural variant of frontotemporal dementia. Brain 2011;134:2456-2477.

3. Snowden J, Neary D, Mann D. Frontotemporal lobar degeneration: clinical and pathological relationships. Acta Neuropathol 2007;114:31-38.

4. Neary D, Snowden JS, Gustafson L, et al. Frontotemporal lobar degeneration: a consensus on clinical diagnostic criteria. Neurology 1998;51:1546-1554.

5. Cardarelli R, Kertesz A, Knebl JA. Frontotemporal dementia: a review for primary care physicians. Am Fam Physician 2010;82:1372-1377.

6. Forman MS, Farmer J, Johnson JK, et al. Frontotemporal dementia: clinicopathological correlations. Ann Neurol 2006;59:952-962.

7. Morris JC, Weintraub S, Chui HC, et al. The Uniform Data Set (UDS): clinical and cognitive variables and descriptive data from Alzheimer disease centers. Alzheimer Dis Assoc Disord 2006;20:210-216.

8. Beekly DL, Ramos EM, Lee WW, et al. The National Alzheimer's Coordinating Center (NACC) database: the Uniform Data Set. Alzheimer Dis Assoc Disord 2007; 21:249-258.

9. Beekly DL, Ramos EM, van Belle G, et al. The National Alzheimer's Coordinating Center (NACC) database: an Alzheimer disease database. Alzheimer Dis Assoc Disord 2004;18:270-277.

10. The National Institute on Aging, and Reagan Institute Working Group on Diagnostic Criteria for the Neuropathological Assessment of Alzheimer's Disease. Consensus recommendations for the postmortem diagnosis of Alzheimer's disease.Neurobiol Aging 1997;18:S1-S2.

11. Kaufer DI, Cummings JL, Ketchel P, et al. Validation of the NPI-Q, a brief clinical form of the Neuropsychiatric Inventory. J Neuropsychiatry Clin Neurosci 2000;12:233-239.

12. Goetz C. Re: UPDRS: status and recommendations. Mov Disord 2004;19:605.

13. Kertesz A, McMonagle P, Blair M, Davidson W, Munoz DG. The evolution and pathology of frontotemporal dementia. Brain 2005;128:1996-2005.

14. Knopman DS, Boeve BF, Parisi JE, et al. Antemortem diagnosis of frontotemporal lobar degeneration. Ann Neurol 2005;57:480-488
15. Mirra SS, Heyman A, McKeel D, et al. The Consortium to Establish a Registry for Alzheimer's Disease (CERAD): part II: standardization of the neuropathologic assessment of Alzheimer's disease. Neurology 1991;41:479-486.

16. Bozeat S, Gregory CA, Ralph MA, Hodges JR. Which neuropsychiatric and behavioural features distinguish frontal and temporal variants of frontotemporal dementia from Alzheimer's disease? J Neurol Neurosurg Psychiatry 2000; 69:178-186.

17. Johnson JK, Head E, Kim R, Starr A, Cotman CW. Clinical and pathological evidence for a frontal variant of Alzheimer disease. Arch Neurol 1999;56:1233-1239.

18. Cairns NJ, Bigio EH, Mackenzie IR, et al. Neuropathologic diagnostic and nosologic criteria for frontotemporal lobar degeneration: consensus of the Consortium for Frontotemporal Lobar Degeneration. Acta Neuropathol 2007;114:5-22.

19. Urwin H, Josephs KA, Rohrer JD, et al. FUS pathology defines the majority of tau- and TDP-43-negative frontotemporal lobar degeneration. Acta Neuropathol 2010;120:33-41.

20. Josephs KA, Hodges JR, Snowden JS, et al. Neuropathological background of phenotypical variability in frontotemporal dementia. Acta Neuropathol 2011;122:137-153.

21. Hodges JR, Davies RR, Xuereb JH, et al. Clinicopathological correlates in frontotemporal dementia. Ann Neurol 2004;56:399-406.

22. Josephs KA, Petersen RC, Knopman DS, et al. Clinicopathologic analysis of frontotemporal and corticobasal degenerations and PSP. Neurology 2006;66:41-48.

23. Goedert M, Ghetti B, Spillantini MG. Frontotemporal dementia: implications for understanding Alzheimer disease. Cold Spring Harb Perspect Med 2012;2:a006254.

24. Josephs KA. Frontotemporal dementia and related disorders: deciphering the enigma. Ann Neurol 2008;64:4-14.

25. Hu WT, Mandrekar JN, Parisi JE, et al. Clinical features of pathologic subtypes of behavioral-variant frontotemporal dementia. Arch Neurol 2007;64:1611-1616.

26. Piguet O, Brooks WS, Halliday GM, et al. Similar early clinical presentations in familial and non-familial frontotemporal dementia. J Neurol Neurosurg Psychiatry 2004; 75:1743-1745.

27. Whitwell JL, Warren JD, Josephs KA, et al. Voxel-based morphometry in tau-positive and tau-negative frontotemporal lobar degenerations. Neurodegener Dis 2004;1:225-230.

\section{Commenting Online is Easier Now with WriteClick ${ }^{\mathrm{TM}}$}

Have a comment on a recent $N e u r o l o g y{ }^{\circledR}$ article you would like to share? Now it is easier and more convenient. Neurology.org has launched WriteClick on the home page and sidebars of each article to encourage remarks and debate among users.

WriteClick is restricted to comments about studies published in Neurology within the last eight weeks.

Learn more at http://www.neurology.org/letters 